dose to human studies is likely to be in appropriate. Nonetheless, we are encouraged by the early lack of evidence for clinically significant effects noted by W are and Isner. Future work must evaluate these effects in other systems and must evaluate the longevity of the observed increases. One disadvantage of monitoring plaque progression in humans is that, unlike animal studies where entire arteries can be excised and quantitatively evaluated in cross-sections, we are limited to more subjective evaluations (leading to use of terms like "perhaps" in describing results) or adverse events which require a large cohort of patients (that is, considerably more than the " 12 patients" mentioned expressly above) followed for a longer periods of time and compared to appropriate controls rather than "historical" or "contemporary" findings culled from the literature. Appropriate evaluations of plaque progression must be undertaken in clinical studies if we are to be certain that we do no harm to our patients in our rush to translate an exciting potential therapy. Finally, effective doses of VEGF must be examined for potential impact on remote plaque formation. Because there are dose-rel ated and model-related effects, we consider it likely that a new therapeutic window will likely emerge.

The prudent response to a cautionary message in a field where apparently contradictory findings have been reported is to carefully select appropriate model systems and eval uate the possible complications in each instance. These issues were previously and appropriately raised by Ware. If significant VEGF-mediated plaque progression does not occur in humans at effective doses, we will breathe a sigh of relief together with the rest of the medical community. At least we as a community will have consciously considered the impact of a relatively complex therapeutic agent.

\section{MichaEL M. DAKE}

Cardiovascular and Interventional Radiology

Stanford University School of Medicine

Stanford, California, USA

Email: mddake@stanford.edu

1. Celletti, F.L. et al. Vascular endothelial growth factor enhances atherosclerotic plaque progression. Nature Med. 7, 425-429 (2001).

2. Van Belle, E et al. Stent endothelialization: time course, impact of local catheter delivery, feasibility of recombinant protein administration, and response to cytokine expedition. Circulation 95, 438-448 (1997).

3. Van Belle, E. et al. Passivation of metallic stents following arterial gene transfer of phVEGF ${ }_{165}$ inhibits thrombus formation and intimal thickening. J. Am. Coll. Cardiol. 29, 1371-1379 (1997).

4. Van Belle, E., Maillard, L., Tio, F.O. \& Isner, J.M Accelerated endothelialization by local delivery of re combinant human vascular endothelial growth factor reduces in-stent intimal formation. Biochem. Biophys. Res. Comm. 235, 311-316 (1997).

5. Asahara, T. et al. Local delivery of vascular endothelial growth factor accelerates reendothelialization and attenuates intimal hyperplasia in balloon-injured rat carotid artery. Circulation 91, 2793-2801 (1995).

6. Asahara, T. et al. Accelerated restitution of endothelial integrity and endothelium-dependent function fol- lowing phVEGF ${ }_{165}$ gene transfer. Circulation 94, 3291-3302 (1996).

7. Tsurumi, Y. et al. Reciprocal relationship between VEGF and NO in the regulation of endothelial integrity. Nature M ed. 3, 879-886 (1997).

8. Hiltunen, M.O et al. Intravascular adenovirus-mediated VEGF-C gene transfer reduces neointima formation in balloon-denuded rabbit aorta. Circulation 102, 2262-2268 (2000).

9. Isner, J.M. et al. Clinical evidence of angiogenesis following arterial gene transfer of phVEGF $_{165}$. Lancet 348, 370-374 (1996).

10. Isner, J.M. Arterial gene transfer for naked DNA for therapeutic angiogenesis: early clinical results. Adv. Drug. Deliv. Rev. 30,185-197 (1998).

11. Vale, P.R. et al. Arterial gene therapy for inhibiting restenosis in patients with claudication undergoing superficial femoral artery angioplasty. Circulation 98, I-66 (1998).

12. Minar, E. et al. Endovascular brachytherapy for prophylaxis of restenosis after femoropopliteal angioplasty: results of a prospective randomized study. Circulation 102, 2694-2699 (2000).

13. Laitinen, $M$. et al. Catheter-mediated vascular endothelial growth factor gene transfer to human coronary arteries after angioplasty. Hum. Gene Ther. 11, 263-270 (2000).

14. Luo, Z., Asahara, T., Tsurumi, Y., Isner, J.M. \& Symes, J.F. Reduction of vein graft intimal hyperplasia and preservation of endothelium-dependent relaxation by topical vascular endothelial growth factor. J. Vasc. Surg. 27, 167-173 (1998).

15. Henry, T.D. et al. Double blind, placebo controlled trial of recombinant human vascular endothelial growth factor-the VIVA trial. J. Am. Coll. Cardiol. 33, 384A (1999)

16. Lazarous, D.F. et al. Comparative effects of basic fibroblast growth factor and vascular endothelial growth factor on coronary collateral development and the arterial response to injury. Circulation 94, 1074-1082 (1996).

17. Moulton, K.S. et al. Angiogenesis inhibitors endostatin or TNP-470 reduce intimal neovascularization and plaque growth in apolipoprotein E-deficient mice. Circulation 99, 1726-1732 (1999).

18. Ware, J.A. Too many vessels? Not Enough? The wrong kind? The VEGF debate continues. Nature Medicine 7, 403-404 (2001).

\title{
Is EDRF a specific marker for TSEs?
}

To the editor-The article by Miele et al. ${ }^{1}$ in the March 2001 issue of Nature Medicine proposed that erythroid differentiation-related factor (EDRF) is a molecular marker of transmissible spongiform encephalopathies (TSEs). They demonstrated a decrease in the expression of EDRF in scrapie-infected mice, and in sheep with scrapie and cattle with bovine spongiform encephalopathies as compared with healthy control animals. The down regulation of EDRF could be detected in blood samples from the infected animals and the authors suggest that EDRF might be useful in non$\mathrm{PrP}^{\mathrm{Sc}}$-based preclinical diagnosis. However, the question of whether the finding is specific to prion-related diseases or related to a nonspecific inhibition of erythropiesis requires further studies, because no disease controls were included, such as animals with various infectious, inflammatory and malignant diseases. An explanation for the result might in fact be a nonspecific inhibition of erythropoiesis. We have demonstrated that interleukins 1$\alpha$ and $1-\beta$, in concentration ranges found in infectious and inflammatory states $^{2-5}$, dose-dependently and selectively suppress the colony formation of the erythroid progenitor cells in bone marrow $^{2}$. As the reduced EDRF levels in TSEs could, as also pointed out by Miele et al., represent a reduction in number of EDRF-expressing progenitors as well as a reduced EDRF expression, the finding might result from a non-specific cytokine-induced inhibition of marrow erythroid progenitors. In support of this view, Kim et al. re- cently reported an induction of mRNAs of proinflammatory cytokines, including interleukins $1-\alpha$ and $1-\beta$, in scrapieinfected mice ${ }^{6}$.

C.P.J. MAURY

Department of Medicine

University of Helsinki

Helsinki, Finland

Email: peter.maury@hus.fi

Miele and Clinton reply-Although we chose to limit speculation on the mechanism underlying our recent findings ${ }^{1}$, we feel that the suggestion by Maury represents one of a number of reasonable interpretations. M aury is correct in stating that variation in the levels of circulating cytokines might affect the numbers of erythroid progenitor cells in bone marrow, although current evi- 